\title{
Disease-modifying properties of long-term lithium treatment for amnestic mild cognitive impairment: randomised controlled trial ${ }^{\dagger}$
}

\author{
Orestes V. Forlenza, Breno S. Diniz, Márcia Radanovic, Franklin S. Santos, Leda L. Talib \\ and Wagner F. Gattaz
}

\section{Background}

Two recent clinical studies support the feasibility of trials to evaluate the disease-modifying properties of lithium in Alzheimer's disease, although no benefits were obtained from short-term treatment.

\section{Aims}

To evaluate the effect of long-term lithium treatment on cognitive and biological outcomes in people with amnestic mild cognitive impairment (aMCl).

\section{Method}

Forty-five participants with $\mathrm{aMCl}$ were randomised to receive lithium $(0.25-0.5 \mathrm{mmol} / \mathrm{l})(n=24)$ or placebo $(n=21)$ in a 12-month, double-blind trial. Primary outcome measures were the modification of cognitive and functional test scores, and concentrations of cerebrospinal fluid (CSF) biomarkers (amyloid-beta peptide $\left(A \beta_{42}\right)$, total tau (T-tau), phosphorylated-tau) (P-tau). Trial registration: NCT01055392.

\section{Results}

Lithium treatment was associated with a significant decrease in CSF concentrations of P-tau $(P=0.03)$ and better perform-ance on the cognitive subscale of the Alzheimer's Disease Assessment Scale and in attention tasks. Overall tolerability of lithium was good and the adherence rate was $91 \%$

\section{Conclusions}

The present data support the notion that lithium has disease-modifying properties with potential clinical implications in the prevention of Alzheimer's disease.

\section{Declaration of interest}

None.
Lithium salts have been extensively used in the past decades for the treatment of bipolar disorder and major depression. Glycogen synthase kinase 3 beta (GSK3B) activity has a central role in the pathophysiology of Alzheimer's disease. ${ }^{1}$ Glycogen synthase kinase 3 beta is the predominant tau-kinase in the brain and modulates the cleavage of amyloid precursor protein. ${ }^{2,3}$ There is substantial support from experimental models that lithium, via inhibition of GSK3B, may modify Alzheimer's disease-specific pathological processes $^{4}$ such as the hyperphosphorylation of tau, ${ }^{5,6}$ the overproduction of the amyloid- $\beta\left(A \beta_{42}\right)$ peptide ${ }^{7,8}$ and $A \beta$-induced neurotoxicity. ${ }^{9}$ Thus, lithium, via the inhibition of GSK3B, may hamper mechanisms that lead to the formation of amyloid plaques and neurofibrillary tangles, and, consequently, having neuroprotective effects against Alzheimer's disease. Indeed, in a previous study we reported that chronic lithium intake was associated with lower prevalence of dementia in older adults with bipolar disorder. ${ }^{10}$ Our findings were confirmed by two additional studies in Denmark with different methodological approaches. ${ }^{11,12}$ Thus, chronic lithium intake may protect against the development of Alzheimer's disease and GSK3B inhibition may be a target for the development of interventions with disease-modifying properties in Alzheimer's disease.

Two clinical trials with lithium were recently conducted in participants with mild and moderate dementia. In an open-label study, lithium carbonate (serum levels $0.3-0.8 \mathrm{mmol} / \mathrm{l}$ ) was administered to 22 people with Alzheimer's disease for up to 1 year. In spite of a high discontinuation rate, side-effects were mild and reversible leading to the conclusion that the prescription of lithium salts for individuals with Alzheimer's disease is relatively

'See editorial, pp. 336-337, this issue. safe. Nevertheless, no cognitive benefits were observed among participants who completed the study. ${ }^{13}$ In a multicentre, singleblind study, participants with mild Alzheimer's disease were treated with lithium sulfate $(0.6-0.8 \mathrm{mmol} / \mathrm{l})$ or placebo for 10 weeks. ${ }^{14}$ Lithium treatment did not have significant effects on cognitive performance and on the cerebrospinal fluid (CSF) concentrations of Alzheimer's disease-related biomarkers.

We hypothesised that these negative results were because of the investigation of lithium in people with clinically manifest Alzheimer's disease, whereas the protective effects of lithium would be better tested during a longer follow-up of individuals at risk of but without dementia, such as people with amnestic mild cognitive impairment (aMCI). ${ }^{15}$ Therefore, the primary objective of this study was to assess the effect of long-term lithium treatment on the progression of cognitive deficits in individuals with aMCI. Secondary objectives were to assess the modification to CSF concentrations of Alzheimer's disease biomarkers (total tau (T-tau), phosphorylated tau (P-tau) and $A \beta_{42}$ ), along with safety and tolerability issues.

\section{Method}

\section{Participant recruitment and study design}

This was a single-centre, randomised, double-blind, placebocontrolled study to assess the potential neuroprotective effects of chronic low-dose lithium treatment in people with aMCI. Participants were community-dwelling out-patients recruited from a cohort dedicated to the study of cognitive ageing at the Institute of Psychiatry, Faculty of Medicine, University of São Paulo. ${ }^{16}$ This study was approved by the local ethical committee and was conducted in adherence with the Helsinki Declaration 
and Good Clinical Practice recommendations; the trial is registered with clinicaltrials.gov: NCT01055392.

Participants were enrolled to this study after signing informed consent. Inclusion criteria were: age 60 years or older; diagnosis of aMCI according to Mayo Clinic criteria ${ }^{15}$ and no evidence of ongoing psychiatric disorders. For individuals with prevalent medical comorbidities requiring continuous pharmacological treatment, enrolment was reliant on the approval of the general practitioner. Figure 1 displays the flow chart for the first 12 months of follow-up. The Appendix outlines the clinical assessment methods and other procedures pertaining to this protocol. All participants were recruited, enrolled, monitored and prescribed by one single physician (O.V.F.) who did not take part in the assessment of baseline or outcome variables. No significant differences in total Cambridge Cognitive Test $(\mathrm{CAMCOG})^{17}$ scores were observed between randomised and non-randomised patients $(88.4$ (s.d. $=5.9)$ and 90.3 (s.d.=3.6) respectively, $P=0.3$ ). The CAMCOG is part of the Cambridge Examination for Mental Disorders in the Elderly (CAMDEX). ${ }^{17}$ It is a brief neuropsychological battery designed to assess global cognitive function and ascertain the impairments that are required for the diagnosis of dementia. Its scores range from 0 to 107 points through 60 items, and it is composed by 8 domains: orientation, memory, language, attention, praxis, perception, abstraction, and calculation.

\section{Lithium dose titration}

Identical tablets containing $150 \mathrm{mg}, 300 \mathrm{mg}, 450 \mathrm{mg}$ or $600 \mathrm{mg}$ of lithium carbonate or placebo were produced at the Central Pharmacy of Hospital das Clínicas, University of São Paulo, and packaged into identical coded blisters. After randomisation, participants started daily doses of $150 \mathrm{mg}$ of lithium, which was titrated to target serum levels of $0.25-0.5 \mathrm{mmol} / \mathrm{l}$ through weekly visits, controlling for tolerability. This range, which is lower than that commonly used for the treatment of affective disorders, was chosen to reduce the risk of discontinuation because of adverse events. Preliminary data from our laboratory showed that treatment with this concentration range for 2 weeks in healthy volunteers caused a 50\% reduction in GSK3B activity in leukocytes (data available from the authors on request). Dispensing was carried out by three pharmacists who did not take part in any other intervention. All participants were instructed to take their prescriptions (lithium or placebo) twice a day at $08.00 \mathrm{~h}$ and $20.00 \mathrm{~h}$, preferentially with meals. If there was any relevant adverse event during the titration phase, the lithium dose was adjusted back to the highest tolerable dose within the treatment range. Serum lithium levels were determined weekly in the titration phase, $12 \mathrm{~h}$ after the last dose. Once stable target levels were achieved, the prescription was maintained until the next visit, which was scheduled at 3-monthly intervals. Any adverse events or modifications in other ongoing prescriptions were to be noted and, if necessary, the lithium treatment re-evaluated.

\section{Outcome variables}

The primary outcomes of this study were the modification of cognitive or functional status and concentrations of amyloid- $\beta_{42}$ peptide $\left(\mathrm{A} \beta_{42}\right)$, total tau (T-tau) and phosphorylated tau (P-tau) in the CSF. The Clinical Dementia Rating (CDR) scale, ${ }^{18}$ including the Sum of Boxes (SoB) score, and the cognitive subscale of the Alzheimer's Disease Assessment Scale (ADAS$\operatorname{Cog})^{19}$ were administered to assess global functional and cognitive state. Memory, attention and executive function were further evaluated with the Consortium to Establish a Registry for Alzheimer's Disease (CERAD) ${ }^{20}$ delayed recall test, Sequence of Letters and Numbers (SLN), ${ }^{21}$ and the Trail Making Test (TMT). ${ }^{22}$ Secondary outcomes were the conversion from aMCI to Alzheimer's disease, as well as safety and tolerability analysis according to longitudinal clinical and laboratory data.

\section{Cerebrospinal fluid biomarkers}

Cerebrospinal fluid samples were obtained by lumbar puncture (L3/L4 or L4/L5 inter-vertebral spaces) between $10.00 \mathrm{~h}$ and $12.00 \mathrm{~h}$, with a 23-gauge needle and using polypropylene tubes. Samples were immediately centrifuged at $3200 \mathrm{~g}$ for $10 \mathrm{~min}$ at $4{ }^{\circ} \mathrm{C}$, and then stored in $500 \mu$ laliquots at $-80^{\circ} \mathrm{C}$ until analysis. The analysis of the CSF concentrations of T-tau, P-tau and $\mathrm{A} \beta_{42}$

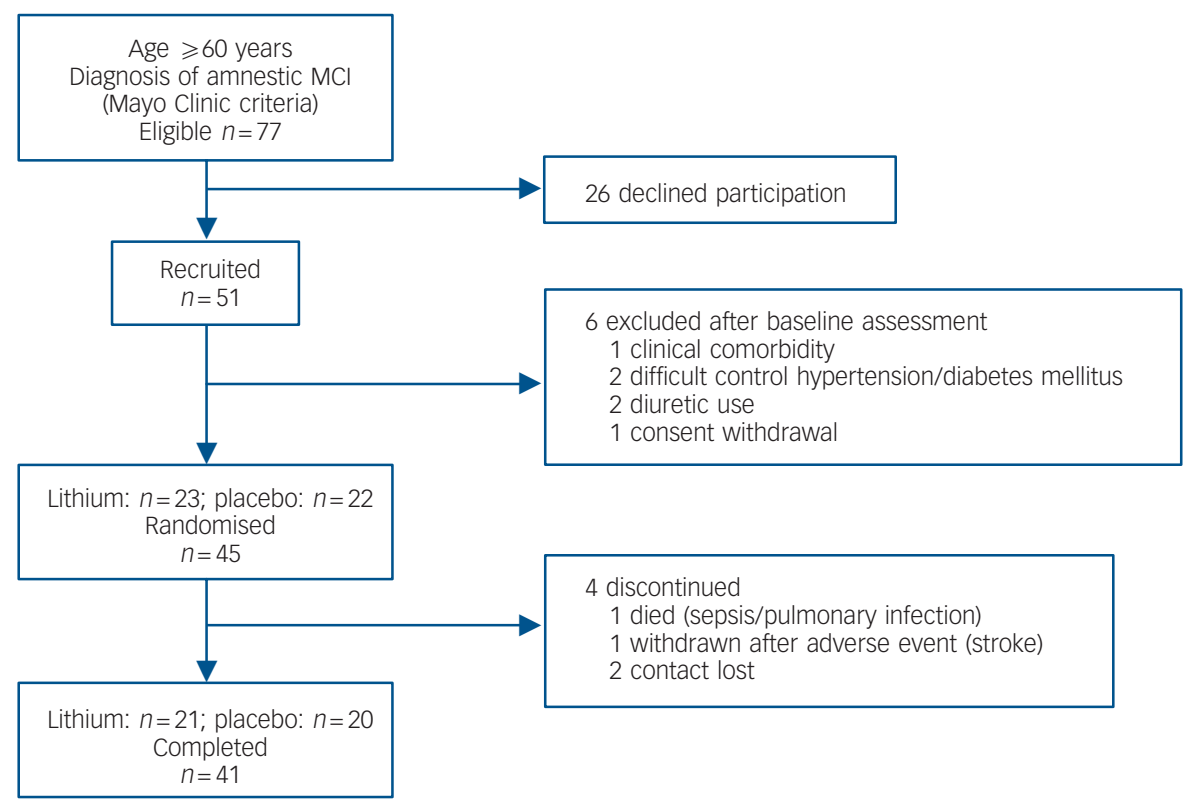

Fig. 1 Study flow chart from recruitment until 12-month follow-up. MCl, mild cognitive impairment. 


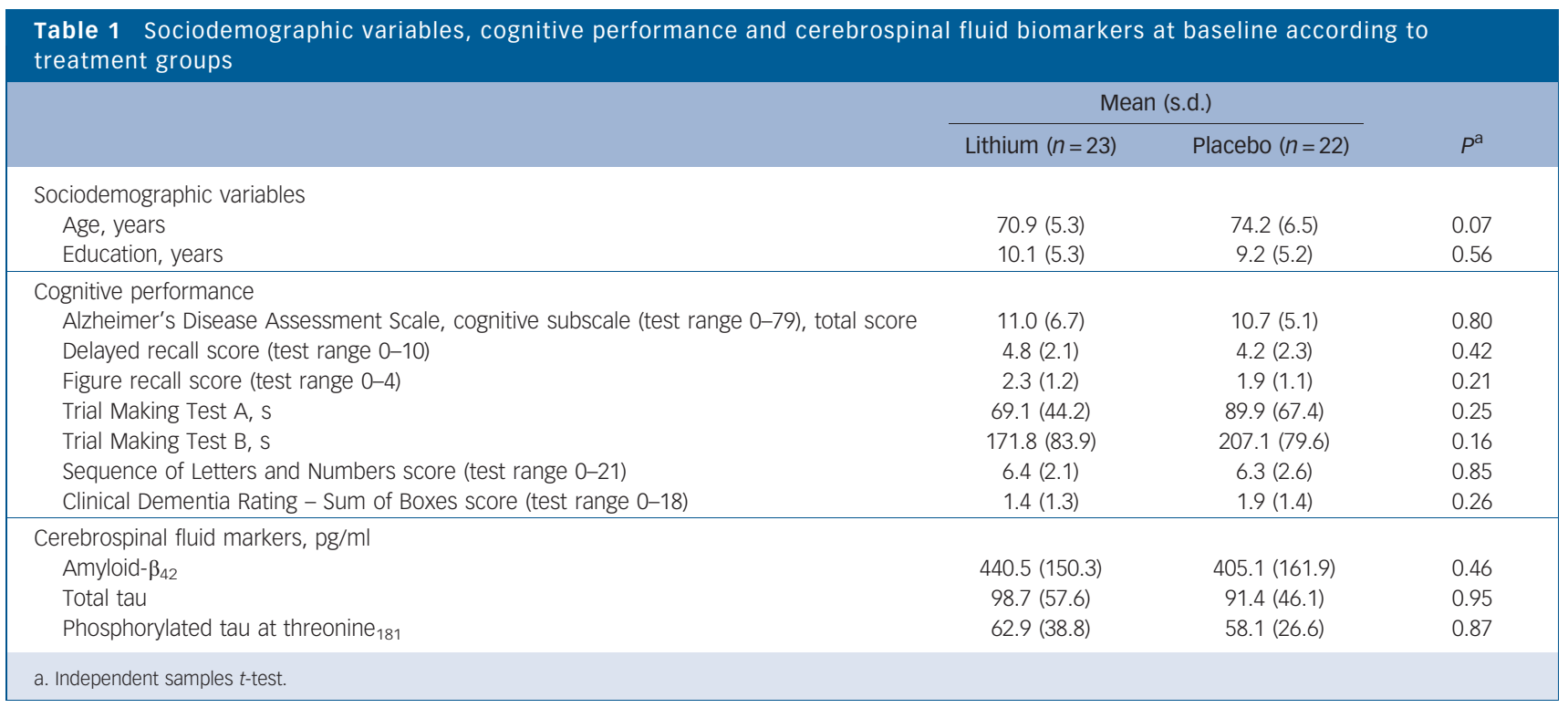

\begin{tabular}{|c|c|c|c|c|c|c|}
\hline & $\begin{array}{l}\text { Lithium baseline } \\
\text { Mean (s.d) }(n=23)\end{array}$ & $\begin{array}{l}\text { Lithium follow-up } \\
\text { Mean (s.d) }(n=21)\end{array}$ & $P^{a}$ & $\begin{array}{l}\text { Placebo baseline } \\
\text { Mean (s.d) }(n=22)\end{array}$ & $\begin{array}{l}\text { Placebo follow-up } \\
\text { Mean (s.d) }(n=20)\end{array}$ & $P^{a}$ \\
\hline \multicolumn{7}{|l|}{ Cognitive performance } \\
\hline $\begin{array}{l}\text { Alzheimer's Disease Assessment Scale, } \\
\text { cognitive subscale (test range 0-79) }\end{array}$ & & $126(66)$ & 021 & 107 (5.1) & $139(85)$ & 0.03 \\
\hline Delayed recall score (test range $0-10$ ), & $4.8(2.1)$ & $4.8(2.2)$ & $\begin{array}{c}0.21 \\
0.4\end{array}$ & $\begin{array}{l}10.7(5.1) \\
4.2(2.3)\end{array}$ & $\begin{array}{l}13.9(8.5) \\
4.5(2.3)\end{array}$ & 0.32 \\
\hline Figure recall score (test range 0-4) & $2.3(1.2)$ & $2.0(1.3)$ & 0.26 & $1.9(1.1)$ & $1.6(1.2)$ & 0.28 \\
\hline Trial Making Test A, S & $69.1(44.2)$ & $62.8(31.5)$ & 0.08 & $89.9(67.4)$ & $63.6(41.9)$ & 0.13 \\
\hline Trial Making Test B, S & $171.8(83.9)$ & $184.9(78.1)$ & 0.58 & $207.1(79.6)$ & $190.7(92.8)$ & 0.25 \\
\hline score (test range 0-18) & $1.4(1.3)$ & $2.2(1.8)$ & 0.03 & $1.9(1.4)$ & $2.8(2.3)$ & 0.04 \\
\hline \multicolumn{7}{|l|}{ Cerebrospinal fluid markers, $\mathrm{pg} / \mathrm{ml}$} \\
\hline Amyloid- $\beta_{42}$ & $440.5(150.3)$ & $391.0(106.1)$ & 0.09 & $405.2(162.0)$ & $424.6(167.4)$ & 0.45 \\
\hline Phosphorylated tau at threonine ${ }_{181}$ & $62.9(39.8)$ & $54.0(40.2)$ & 0.15 & $58.1(26.6)$ & $63.7(29.5)$ & 0.07 \\
\hline Total tau & $98.7(57.6)$ & $109.4(79.5)$ & 0.18 & $91.4(46.2)$ & $100.4(51.9)$ & 0.09 \\
\hline
\end{tabular}

were done in duplicate with the INNo-Bia AlzBio3 assay (Innogenetics, Belgium), a multiplexed, microsphere-based method for xMAP-Luminex platform allowing the simultaneous analysis of all biomarkers in the same aliquot.

After pre-wetting of the filter plate with a wash buffer, a suspension of microspheres carrying the corresponding capturing antibodies (AT120 for T-tau, AT270 for P-tau phosphorylated at Threonine $_{181}$, and $4 \mathrm{D} 7 \mathrm{~A} 3$ for $A \beta_{42}$ ) was added to the plate. A mixture of biotinylated detection monoclonal antibodies, designed to specifically recognise one of the capturing antibodies (HT7 for T-tau and P-tau, and 3D6 for $A \beta_{42}$ ), and $75 \mu$ l of CSF aliquots or standards were added to the plate and incubated overnight, protected from light. The following morning, the plate was washed and a detection conjugate (phycoerythrin-labelled streptavidin) was added and incubated for $1 \mathrm{~h}$ at room temperature. A reading solution (phosphate buffer saline) was added to the plate after a final wash and the assay was analysed on a Luminex 100 IS platform (Luminex, Austin, USA). Standard curves were constructed for each biomarker using a sigmoid curve fitting method, and mean fluorescence values for duplicate samples were used to yield CSF concentrations of T-tau, P-tau and $\mathrm{A} \beta_{42}$ in $\mathrm{pg} / \mathrm{ml}$ of CSF.

\section{Statistical analysis}

Chi-squared and Fisher's exact tests were carried out to assess differences in categorical data (gender distribution and conversion status) between lithium and placebo groups. Student's $t$-tests (independent samples) were carried out to assess mean differences in baseline clinical, cognitive and biological variables between treatment groups and between the subsets of participants who progressed to Alzheimer's disease as opposed to those who remained stable. Paired sample $t$-tests were carried out to address differences in the cognitive and biological variables between baseline and the 1-year follow-up within-treatment groups. Non-parametric tests (Mann-Whitney $U$-test and Wilcoxon rank test) were further carried out for analysis of biological data in subanalyses with limiting sample sizes. 
Table 3 Concentrations of cerebrospinal fluid biomarkers at baseline and after 12 months of treatment with lithium or placebo and according to conversion status

\begin{tabular}{|c|c|c|c|}
\hline \multirow[b]{2}{*}{ Cerebrospinal fluid biomarkers, pg/ml } & \multicolumn{2}{|c|}{ Mean (s.d.) } & \multirow[b]{2}{*}{$P^{a}$} \\
\hline & Baseline & Follow-up & \\
\hline \multicolumn{4}{|l|}{ Lithium group } \\
\hline \multicolumn{4}{|l|}{ Remained stable $(n=16)$} \\
\hline Amyloid- $\beta_{42}$ & $477.2(139.5)$ & 411.9 (104.9) & 0.06 \\
\hline Total tau & $80.1(39.8)$ & $88.27(61.6)$ & 0.39 \\
\hline Phosphorylated tau at threonine 181 & $48.3(16.6)$ & $40.7(16.6)$ & 0.006 \\
\hline \multicolumn{4}{|l|}{ Progressed to Alzheimer's disease $(n=4)$} \\
\hline Amyloid- $\beta_{42}$ & $269.1(26.7)$ & $293.4(31.0)$ & 0.60 \\
\hline Total tau & $185.6(50.0)$ & $207.9(90.2)$ & 0.60 \\
\hline Phosphorylated tau at threonine ${ }_{181}$ & $130.8(42.6)$ & $115.9(64.3)$ & 0.90 \\
\hline \multicolumn{4}{|l|}{ Placebo group } \\
\hline \multicolumn{4}{|l|}{ Remained stable $(n=13)$} \\
\hline Amyloid- $\beta_{42}$ & $430.0(169.8)$ & $455.2(169.3)$ & 0.64 \\
\hline Total tau & $80.5(44.2)$ & $86.1(42.6)$ & 0.50 \\
\hline Phosphorylated tau at threonine 181 & $50.4(21.9)$ & $57.2(28.5)$ & 0.03 \\
\hline \multicolumn{4}{|l|}{ Progressed to Alzheimer's disease $(n=7)$} \\
\hline Amyloid- $\beta_{42}$ & $324.3(114.1)$ & $325.1(132.5)$ & 0.71 \\
\hline Total tau & $126.5(37.2)$ & $146.9(57.6)$ & 0.07 \\
\hline Phosphorylated tau at threonine ${ }_{181}$ & $80.9(29.2)$ & $83.1(26.4)$ & 0.84 \\
\hline
\end{tabular}

\section{Results}

\section{Baseline assessment}

No differences were found between those participants treated with lithium and those treated with placebo regarding sociodemographic, cognitive and biological variables prior to treatment (Table 1).

\section{Outcome (12-month) assessment}

Forty-one participants (91\% of the total sample) completed 1 year of follow-up, with similar representation in the lithium $(n=21)$ and placebo $(n=20)$ groups $\left(\chi^{2}=0.178, P=0.52\right)$. Four participants discontinued treatment within 12 months (Fig. 1). There were no significant differences in sociodemographic data, cognitive variables and CSF biomarkers between completers and non-completers (data not shown). Eleven participants (24\% of the original sample) progressed to Alzheimer's disease after 12 months of follow-up. As expected, these individuals displayed at baseline the typical 'Alzheimer's disease signature' in the CSF, i.e. higher concentrations of T-tau and P-tau and lower concentrations of $A \beta_{42}$, as compared with non-converters (data not shown). The number of conversions was higher in the placebo group (7/20) compared with the lithium group (4/21), but this difference was not significant (Fisher's, $P=0.2$ ). All participants irrespective of treatment with lithium or placebo, had a slight but significant worsening of global functional state, as indicated by mean CDR-SoB scores $(P<0.04)$. However, the magnitude of cognitive decline was smaller in the participants treated with lithium than in the placebo group, as shown by the modification of the ADAS-Cog and in the SLN test scores (Table 2).

The participants treated with lithium had a decrease in the concentrations of P-tau (end-point minus baseline values: $-8.9 \mathrm{pg} / \mathrm{ml}$, s.d. $=24.3$ ), whereas a slight increase was observed in participants who received placebo $(5.6 \mathrm{pg} / \mathrm{ml}$, s.d. $=11.4)$; this difference was significant $(P=0.02)$. No such effect was observed for the concentrations of T-tau (Fig. 2, Table 2). Per definition, the participants who converted to dementia in both treatment groups had an increase in the CDR-SoB scores, as opposed to those who remained stable. However, this cognitive and functional worsening in those who progressed to Alzheimer's disease was attenuated by lithium (baseline: 3.3 (s.d.=1.3), endpoint: 4.4 (s.d. $=1.5), P=0.3$ ) as compared with placebo (baseline: 3.4 (s.d.=1.4), end-point: 5.6 (s.d.=1.5), $P=0.03$ ). Table 3 displays the modifications in CSF biomarkers in four subsets of participants: those who remained stable and were receiving lithium; those who progressed to Alzheimer's disease receiving lithium; those who remained stable and were receiving placebo; and those who progressed to Alzheimer's disease receiving placebo. The reduction of CSF P-tau in those treated with lithium occurred only in the participants who remained stable over time $(P=0.006)$, whereas no significant reduction was found in those treated with lithium who progressed to Alzheimer's disease $(P=0.90)$. In the placebo group both those who were stable and those who progressed to Alzheimer's disease had a slight increase in P-tau. Irrespective of treatment groups and conversion status, no significant changes were noted in the concentrations of T-tau and $\mathrm{A} \beta_{42}$.

\section{Safety analysis}

Forty-three individuals were included in the safety analysis. General tolerability was good given the high adherence rate (91\%) at 1 year (Fig. 1). The occurrence of side-effects was similar in the lithium- (58\%) and placebo-treated (42\%) groups $\left(\chi^{2}=2.17, P=0.13\right)$. Most of the adverse events were mild and transient, and involved the gastrointestinal system, and no action was required. Three participants in the placebo group had side-effects of moderate intensity (nausea and hand tremor) and dose reduction was sufficient to relieve complaints. One person in the lithium group presented with a major cerebrovascular event (ischemic stroke) during treatment and was withdrawn from the study. One participant in the placebo group died during the study due to sepsis secondary to pneumonia. These serious adverse events were considered unrelated to the studied drug.

\section{Discussion}

\section{Main findings}

This is the first double-blind, placebo-controlled study addressing the protective properties of lithium in people with aMCI. We 


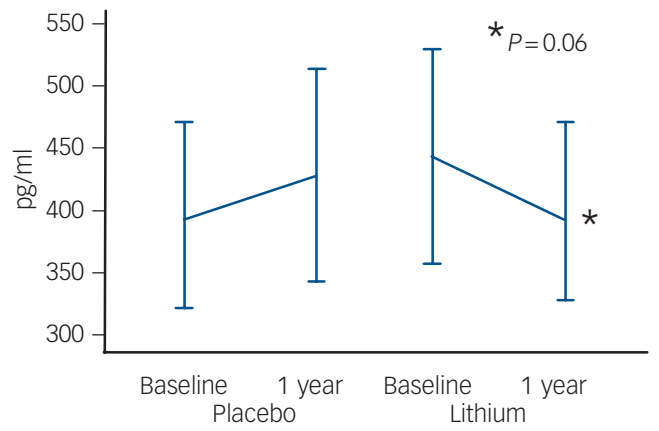

(b)

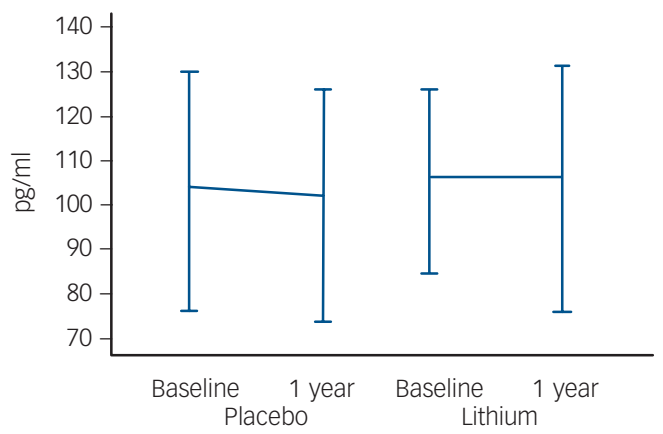

(c)

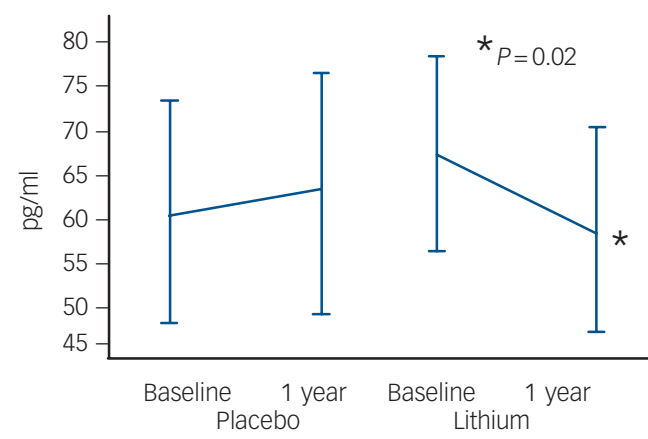

Fig. 2 Modification of cerebrospinal fluid biomarkers at follow-up according to treatment groups. (a) Amyloid- $\beta_{42}$, (b) total tau, (c) phosphorylated tau.

found that lithium treatment for a year reduced the cognitive decline, as compared with placebo. Moreover, long-term lithium treatment was associated with a significant reduction in CSF concentration of P-tau. It should be stressed that lithium treatment was safe and well-tolerated at serum concentrations of $0.25-0.5 \mathrm{mmol} / \mathrm{l}$.

\section{Other studies}

Two previous studies failed to find a significant effect of lithium either on cognition or in Alzheimer's disease-related biomarkers. ${ }^{13,14}$ Possible reasons were the higher side-effects and drop-out rates associated with higher lithium levels; the shorter follow-up periods; and the inclusion of people in more advanced stages of cognitive deterioration, i.e. mild and moderate Alzheimer's disease. In contrast, the present study was designed in concordance with two recent consensus papers addressing methodological aspects of trials on disease-modification in Alzheimer's disease. ${ }^{23,24}$ Accordingly, such trials should start as early as possible in the disease continuum to warrant clinically relevant benefits, and should preferentially include biological information for the definition of cases and/or outcomes. From that viewpoint, people with incipient or prodromal Alzheimer's disease will more likely benefit from disease modification than those with clinically manifest dementia. Thus, the strengths of the present study are: the inclusion of individuals without dementia but with aMCI; the determination of baseline and end-point biomarkers; the long duration of follow-up; and the low-dose lithium regimen, yielding a low drop-out rate.

\section{Implications}

In the present study, lithium treatment reduced CSF P-tau in participants with aMCI who did not convert to Alzheimer's disease. Since increased P-tau is a specific marker of the pathological process in Alzheimer's disease, it is tempting to speculate that the reduction of the concentrations of P-tau in the CSF may be a useful parameter to predict the preventive effects of lithium regarding the conversion from mild cognitive impairment to Alzheimer's disease. ${ }^{25}$

We further hypothesise that the disease-modifying effect of lithium in Alzheimer's disease may be stage-dependent. Among converters, baseline P-tau and $A \beta_{42}$ levels were similar to those found in clinically manifest Alzheimer's disease, suggesting that the more severe homeostatic imbalance is less responsive to lithium. In contrast, individuals with less severe deficits (i.e. individuals with mild cognitive impairments who remained stable) may benefit most from the neurobiological effects of lithium as they are at earlier stages in the disease continuum. If replicated in future studies, we believe that the understanding of this dissociated effect of lithium according to the magnitude of intracerebral pathology may have important clinical implications, particularly referring to the question of when to start treatment with antidementia drugs. Presumably, maximum benefits from treatment are subject to the early diagnosis of Alzheimer's disease, preferentially at mild cognitive impairment stages or earlier, when the pathological changes are still incipient and the restoration of normal physiological status is more likely to occur. Our data, together with evidence from the literature discussed, suggest that long-term lithium treatment at relatively lower serum levels may be a safe and inexpensive strategy to prevent, or at least to delay the progression from pre-dementia stages to clinical Alzheimer's disease.

In conclusion, the present findings reinforce the notion that in an individual at risk for Alzheimer's disease, lithium may have a protective effect on the progression of cognitive impairment to dementia. This is probably a consequence of the effect of lithium on $\mathrm{GSK}_{3} \mathrm{~B}^{26}$ and possibly on other pivotal cascades involved in the pathophysiology of Alzheimer's disease. We acknowledge that the relatively small sample size of this single-centre study is a limitation to the generalisation of the current findings. Therefore, we think that the present results warrant replication in multicentric trials with a larger sample.

Orestes v . Forlenza, $\mathrm{MD}, \mathrm{PhD}$, Breno $\mathrm{S}$. Diniz, MD, Márcia Radanovic, $\mathrm{MD}$, $\mathrm{PhD}$ Franklin S. Santos, MD, PhD, Leda L. Talib, BSc, Wagner F. Gattaz, MD, PhD, Laboratory of Neuroscience (LIM 27), Department and Institute of Psychiatry, Faculty of Medicine, University of São Paulo, São Paulo, Brazil

Correspondence: Orestes V. Forlenza, MD, PhD, Laboratory of Neuroscience (LIM 27) Department and Institute of Psychiatry, Faculty of Medicine, University of São Paulo, Rua Dr. Ovídio Pires de Campos 785, 05403-010 - São Paulo, SP, Brazil. Email: forlenza@usp.br

First received 17 Mar 2010, final revision 2 Jun 2010, accepted 10 Jun 2010 


\section{Funding}

The present work was supported by Conselho Nacional de Pesquisa Cientifica (CNPq Project 554535/2005-0), Alzheimer's Association (NIRG-08-90688) and Fundação de Amparo à Pesquisa do Estado de São Paulo (FAPESP, Project 02/13633-7). The Laboratory of Neuroscience (LIM-27) receives financial support from Associação Beneficente Alzir Denise Hertzog da Silva (ABADHS).

\section{Acknowledgements}

We are indebted to the pharmacists Aaron Barbosa, Maria F. Castanheira and Severiano Tolentino de Freitas Neto for their contribution to this study dispensing trial drugs.

\section{Appendix}

\section{Clinical examination, cognitive assessment and laboratory procedures}

Physical and neurological

examination

Cognitive assessment

Alzheimer's Disease

Assessment Scale -

cognitive subscale

Sequence of Letters and

Numbers

Trail Making Test $\mathrm{A}$ and $\mathrm{B}$

Consortium to Establish a

Registry for Alzheimer's

Disease: word-list recall

and figure recall

Functional assessment:

Clinical Dementia Rating

Scale

Side-effects: the UKU

side-effect rating scale ${ }^{27}$

Monitoring and dose titration:

lithium levels

Biochemistry and biomarkers:

blood sampling

Cerebral spinal fluid sampling

for biomarkers: lumbar

puncture

6 Lovestone S, Davis DR, Webster MT, Kaech S, Brion JP, Matus A, et al. Lithium reduces tau phosphorylation: effects in living cells and in neurons at therapeutic concentrations. Biol Psychiatry 1999; 45: 995-1003.

7 Phiel CJ, Wilson CA, Lee VM, Klein PS. GSK-3alpha regulates production of Alzheimer's disease amyloid-beta peptides. Nature 2003; 423: 435-9.

8 Rockenstein $\mathrm{E}$, Torrance $\mathrm{M}$, Adame A, Mante M, Bar-on P, Rose JB, et al. Neuroprotective effects of regulators of the glycogen synthase kinase-3 $\beta$ signaling pathway in a transgenic model of Alzheimer's disease are associated with reduced amyloid precursor protein phosphorylation. J Neurosci 2007; 27: 1981-91.

9 Koh SH, Noh MY, Kim SH. Amyloid- $\beta$ induced neurotoxicity is reduced by inhibition of glycogen synthase kinase-3. Brain Res 2008; 1188: 254-62.

10 Nunes PV, Forlenza OV, Gattaz WF. Lithium and risk for Alzheimer's disease in elderly patients with bipolar disorder. Br J Psychiatry 2007; 190: 359-60.

11 Kessing LV, Søndergård L, Forman JL, Andersen PK. Lithium treatment and risk of dementia. Arch Gen Psychiatry 2008; 65: 1331-5.

12 Kessing LV, Forman JL, Andersen PK. Does lithium protect against dementia? Bipolar Disord 2010; 12: 87-94.

13 Macdonald A, Briggs K, Poppe M, Higgins A, Velayudhan L, Lovestone S. A feasibility and tolerability study of lithium in Alzheimer's disease. Int J Geriatr Psychiatry 2008; 23: 704-11.

14 Hampel H, Ewers $M$, Bürger $K$, Annas $P$, Mörtberg $A$, Bogstedt $A$, et al. Lithium trial in Alzheimer's disease: a randomized, single-blind, placebocontrolled, multicenter 10-week study. J Clin Psychiatry 2009; 70: 922-31.

15 Petersen RC, Smith GE, Waring SC, Ivnik RJ, Tangalos EG, Kokmen E. Mild cognitive impairment: clinical characterization and outcome. Arch Neurol 1999; 56: 303-8.

16 Diniz BS, Nunes PV, Yassuda MS, Pereira FS, Flaks MK, Viola LF, et al. Mild cognitive impairment: cognitive screening or neuropsychological assessment? Rev Bras Psiquiatr 2008; 30: 316-21.

17 Roth M, Tym E, Mountjoy CQ, Huppert FA, Hendrie H, Verina S, et al. CAMDEX. A standardised instrument for the diagnosis of mental disorder in the elderly with special reference to the early detection of dementia. Br J Psychiatry 1986; 149: 698-709.

18 Morris JC. The Clinical Dementia Rating (CDR): current version and scoring rules. Neurology 1993; 43: 2412-4.

19 Rosen WG, Mohs RC, Davis KL. A new rating scale for Alzheimer's disease. Am J Psychiatry 1984; 141: 1356-64.

20 Morris JC, Mohs RC, Rogers H, Fillenbaum G, Heyman A. Consortium to Establish a Registry for Alzheimer's Disease (CERAD) clinical and neuropsychological assessment of Alzheimer's disease. Psychopharmacol Bull 1988; 24: 641-52

21 Wechsler D. Wechsler Adult Intelligence Scale - Third Edition, WAIS-III. The Psychological Corporation, 1997.

22 Reitan RM. Halstead-Reitan Neuropsychological Test Battery. Reitan Neuropsychology Laboratory Press, 1985.

23 Vellas B, Andrieu S, Sampaio C, Wilcock G, et al. Disease-modifying trials in Alzheimer's disease: a European task force consensus. Lancet Neurol 2007; 6: $56-62$.

1 Hooper C, Killick R, Lovestone S. The GSK3 hypothesis of Alzheimer's disease J Neurochem 2008; 104: 1433-9.

2 Ishiguro K, Shiratsuchi A, Sato S, Omori A, Arioka M, Mobayashi S, et al. Glycogen synthase kinase $3 \beta$ is identical to tau protein kinase I generating several epitopes of paired helical filaments. FEBS Lett 1993; 325: 167-72.

3 Muyllaert D, Kremer A, Jaworski T, Borghgraef $P$, Devijver $H$, Croes $S$, et al Glycogen synthase kinase-3 3 , or a link between amyloid and tau pathology? Genes Brain Behav 2008; 7 (suppl 1): 57-66.

4 Chuang DM, Manji HK. In search of the holy grail for the treatment of neurodegenerative disorders: has a simple cation been overlooked? Biol Psychiatry 2007; 62: 4-6.

5 Hong M, Chen DC, Klein PS, Lee VM. Lithium reduces tau phosphorylation by inhibition of glycogen synthase kinase-3. J Biol Chem 1997; 272: 25326-32.

24 Vellas B, Andrieu S, Sampaio C, Coley N, Wilcock G, et al. Endpoints for trials in Alzheimer's disease: a European task force consensus. Lancet Neurol 2008; 7: 436-50.

25 Diniz BS, Pinto Júnior JA, Forlenza OV. Do CSF total tau, phosphorylated tau, and beta-amyloid 42 help to predict progression of mild cognitive impairment to Alzheimer's disease? A systematic review and meta-analysis of the literature. World J Biol Psychiatry 2008; 9: 172-82.

26 Bhat RV, Budd Haeberlein SL, Avila J. Glycogen synthase kinase 3 : a drug target for CNS therapies. J Neurochem 2004; 89: 1313-7.

27 Lingjaerde O, Ahlfors UG, Bech P, Dencker SJ, Elgen K. The UKU side effect rating scale. A new comprehensive rating scale for psychotropic drugs and a corss-sectional study of side effects in neuroleptic-treated patients. Acta Psychiatr Scand 1987; 334 (suppl): 1-100. 